\title{
El tercer maestro: el espacio natural como catalizador para una educación ambiental efectiva
}

\author{
The Third Teacher: Natural Space as a Catalyst for Effective \\ Environmental Education
}

Franklin Castillo-Retamal ${ }^{1}$; Fernanda Cordero-Tapia ${ }^{2}$

\begin{abstract}
RESUMEN
Hoy nos enfrentamos una situación y condición ambiental afectada por la acción antrópica en todos sus niveles, la cual, de cierta forma, se ha normalizado. Si bien existen acciones tendientes a aminorar los daños provocados, es necesario un esfuerzo mayor por alcanzar la autonomía de pensamiento y la libertad de acción para actuar frente al escenario actual. La educación ambiental y la educación para el desarrollo sustentable intentan, desde sus postulados, acercar u orientar esos procesos y hacerlos parte del acto humano. Vale decir, normalizar el cuidado, preservación y protección ambiental. Desde allí, la arquitectura natural del paisaje, se torna una sala de clases siempre abierta y acogedora, en la que se entrelazan estímulos para el aprendizaje valioso y permanente.
\end{abstract}

Palabras claves: educación ambiental; espacio natural; desarrollo sustentable; conciencia planetaria; medioambiente.

\begin{abstract}
Today we face an environmental situation and condition affected by the anthropic action at all levels, which, in a way, has been normalized. Although there are actions aiming to reduce the damage, a greater effort is needed to achieve autonomy of thought and freedom of action to act on the current scenario. Environmental education and education for sustainable development try, from their postulates, to approach or guide these processes and make them part of the human act. That is, to normalize environmental care, preservation and protection. From there, the natural landscape architecture becomes a classroom that is always open and welcoming, in which stimuli for valuable and permanent learning are intertwined.
\end{abstract}

Keywords: Environmental education; natural space; sustainable development; planetary awareness; environment.

1 Académico de la Facultad de Ciencias de la Educación, Universidad Católica del Maule, Talca, Chile; fcastillo@ucm.cl.

2 Académica de la Escuela de Educación Física, Universidad Católica del Maule, Talca, Chile; fdacorderotapia@gmail.com. 


\section{Introducción}

Desde el inicio de los tiempos, los humanos han tenido una relación estrecha con el medio en el que viven y conviven, usufructuando de lo que la tierra provee en intensa y permanente inter-relación, además de interactuar con absoluto sentido del derecho de servirse y explotar la misma. Sin embargo, esta actitud ha estimulado un aceleramiento en el proceso de degradación del medio ambiente, trayendo con eso, la acción perniciosa de su estancia. Una de las causas de este deterioro y actitud poco amigable se debe, probablemente, al desarrollo social, científico y tecnológico, las estructuras políticas y el estilo de vida del mundo moderno basado en la cultura del usar y botar, causas que han provocado, lamentablemente, la situación de deterioro actual del planeta.

Sin embargo, en las últimas décadas se han alcanzado algunos acuerdos, tratados y pactos internacionales que tienen como norte responder de manera satisfactoria a la necesidad de enfrentar la problemática ambiental; no obstante, estos esfuerzos no han tenido los resultados esperados (Castillo-Retamal y Cordero-Tapia, 2019).

En este sentido, la educación ambiental se transforma en una herramienta esencial toda vez que, a través de sus planteamientos y acciones, permite generar cambios actitudinales, conductuales y de hábitos en los individuos (Tikka, Kuitunen y Tynys, 2000; Sosa, IsaacMárquez, Eastmond, Ayala y Arteaga, 2010; Fuentealba, 2011, 2018; Mendieta y Gutiérrez, 2014).

En esta misma línea, Fuentealba, Marín, Castillo y Roco (2017), plantean que los cambios de hábito y conductuales deben ser incorporados a partir de los objetivos de la educación ambiental, a través de la enseñanza formal y no formal, de tal manera que estos procesos educativos contribuyan al cambio y transformación social. No obstante, estos deben estar asociados a la vivencia, de tal manera que cada aprendizaje cobre sentido en tanto conocedor del fenómeno, vale decir, no será posible un conocimiento pleno y no será posible saber sin estar con contacto con la materia viva que entrega detalles desde su propia existencia (Castillo, 2011). 


\section{Aprender con y desde el sentido: posibilidades pedagógicas de la educación ambiental}

Según Mejía (2016), la educación ambiental debe dinamizar y permitir las inter-relaciones en un espacio para representarnos a través de tres esferas, ámbitos o dimensiones que, como indica además Sauvé (2004), serían: 1) relación consigo mismo, 2) relación con el otro y 3) relación con el oikos (en griego significa casa), permitiendo así el reconocimiento del ser y la sociedad, como así también la aceptación de los diferentes conocimientos, de tal forma que se fomente una identidad cultural y un diálogo de saberes (Leff, 2006). Siendo así, toma con mayor fuerza la idea de un trabajo inter/multi/transciplinar para abordar de manera integral este proceso educativo, alejándonos del activismo teórico ambiental para centrarnos en la episteme que sustenta su propuesta. Con este planteamiento, se proponen las actividades en los espacios naturales como una oportunidad para el reconocimiento personal (el yo), del otro y lo otro (oikos), de tal forma de posibilitar la generación de una ética ambiental asumiendo que el hombre como especie no está ajeno a la naturaleza, sino que es parte de la misma (Montserrat, 2019) y, por lo tanto, debe ser responsable de identificar los problemas y proponer soluciones para mejorar el espacio en el que co-habita.

En esta línea Tréllez (2006), plantea que es necesaria la renovación e innovación en educación ambiental, de tal forma de buscar otros caminos que identifiquen las expectativas y requerimientos, a partir del diálogo entre saberes. Vale decir, un tratamiento sistémico donde los conceptos ambientales sean extendidos y valorados epistemológica, ontológica y metodológicamente. Para ello, esta acción dialógica permitiría la re-significación y recontextualización de los procesos pedagógicos e investigativos, como así también la reconfiguración del sentido de los mismos.

La plataforma que ofrece la educación ambiental para esta significación, permitiría aproximarnos a la construcción de sentido que, según Frankl (2000), es una toma de conciencia de la realidad y se constituye a partir de las propias vivencias y relaciones, entendiendo también que esta visión difiere de un individuo a otro, así como de un día para otro. O sea, lo importante aquí no es el sentido tratado en términos generales, sino el significado concreto que cada individuo le otorga en cada etapa de su vida a los espacios y 
lugares que visita. Desde esta mirada, los procesos pedagógicos en educación ambiental deben ser tratados como un instrumento de trasformación social, como vía para generar cambios frente a las dificultades ambientales y para la formación de ciudadanos conscientes del mundo que los rodea (Strobl, 2005; Pérez, Pérez y Quijano, 2009; Juyent y Kong, 2011). Para tal fin, las aulas abiertas y esta necesidad de estar en contacto directo con la materia, se presentan como el espacio y estrategia para alcanzar estos objetivos.

En estos términos, las vivencias y la experiencia profunda, resultan determinantes al momento de adquirir aprendizajes, situación que es posible soslayar a partir de planteamientos metodológicos que apunten no solo a la incorporación conceptual y trabajo cognitivo, sino que a la modificación de conductas y hábitos que permanezcan en el diario vivir de cada individuo.

El aprendizaje con sentido, apartado de la mecánica técnica y estructura convencional, emerge a partir del compartir experiencias y vivencias y, en la medida en que ellas se absorban, analicen y expliquen el aprendizaje, es posible alcanzar la autonomía del pensamiento, por tanto, del propio conocimiento (Castillo, 2011).

Esta mirada más amplia del tratamiento y abordaje educativo, se condice de alguna manera con lo planteado por Castillo-Sarmiento, Suárez-Gélvez y Mosquera-Téllez (2017), quienes indican que la postura paradigmática asumida a partir de mediados del siglo XX describe la relación ser humano-naturaleza desde una óptica de la sociedad de la información, con una concepción sistémica-interactiva, y otra, desde la conjunción social-natural-cultural, a partir de una concepción sistémica-sostenible-local, lo que presume un tratamiento y abordaje complejo del fenómeno.

En tal sentido, la educación cumple un rol esencial en la transición social hacia el desarrollo sustentable, toda vez que permite comprender conceptos asociados a la sustentabilidad, ser conscientes del impacto sobre el medio ambiente a partir de las acciones humanas, y desarrollar y potenciar habilidades para modificar e interactuar con el entorno.

La educación ambiental y la educación para el desarrollo sustentable, se unen para abordar los principales temas que afectan los cambios globales que hoy enfrenta la humanidad 
(Ministerio del Medio Ambiente, 2018a, b). En este sentido, la educación ambiental permite formar y educar personas a partir de los 4 pilares fundamentales de la educación -a saber: aprender a conocer, aprender a hacer, aprender a vivir juntos y con los demás, y aprender a ser (Delors, 2010)- y, desde la educación para el desarrollo sustentable, mantener el respeto y buenas prácticas para con el entorno y los seres vivientes, desde el accionar individual como también desde el colectivo, de tal forma de aminorar el impacto sobre el medio natural.

La Ley General de Educación (Ley 20.370, 2009) y las Bases Curriculares para la Enseñanza Básica (Decreto Supremo N433, 2012) y educación media, entregan orientaciones para incorporar la educación ambiental y un sinnúmero de oportunidades que permiten abordar las temáticas ambientales que son fundamentales para el desarrollo sustentable del país. En esta línea, es necesario realizar mayores esfuerzos para integrar los temas ambientales de manera transversal y con un enfoque territorial, de manera que el proceso de enseñanza y aprendizaje sea pertinente, contextualizado y significativo. Sin embargo, se requiere mirar estas temáticas desde un enfoque interdisciplinar que genere la formación de una ciudadanía que tenga el conocimiento y actitudes para enfrentar los desafíos ambientales globales y locales (Ministerio del Medio Ambiente, 2018a, b), por lo que los procesos educativos deben ser un espacio donde los/as estudiantes se conviertan en los protagonistas de su aprendizaje, generando conocimientos, adquiriendo valores, fortaleciendo actitudes y desarrollando habilidades, en virtud de transformarse en agentes de cambio y responsablemente activos/as frente a sus actos cotidianos.

En esta línea, el/la docente es uno de los agentes más relevantes en la orientación de los aprendizajes en la etapa escolar, desde el punto de vista del abordaje de temáticas asociadas a la educación ambiental y la educación para el desarrollo sustentable. El o la docente no se considera como un emisor de conocimientos, sino que se convierte en un agente sensibilizador, capaz de provocar cambios de conductas y hábitos hacia el medio ambiente en los estudiantes (Fuentealba, 2011; Eslava-Zapata, Zambrano-Vivas, Chacón-Guerrero, González-Júnior y Martínez-Nieto, 2018; Castillo-Retamal y Cordero-Tapia, 2019). No obstante lo anterior, se evidencia una inadecuada preparación en la formación inicial de los/as docentes, lo que eventualmente sería una de las principales causas asociadas a la ¿deficiente?/¿insuficiente? implementación de la educación ambiental en los 
establecimientos educacionales (Agüero et. al., 2011; Muñoz-Pedreros, 2014; Vendrasco, Guzmán, Carrasco y Merino, 2018).

Esto último, en el caso que se mejore o corrija, es lo que permitiría dar posibilidades a los/as estudiantes de desarrollar interés sobre temáticas ambientales, lo que implica llevar a cabo, por parte del docente, una variedad de actividades didácticas que motiven a sus estudiantes a resolver situaciones asociadas a problemas ambientales de los que son parte. Ello significa reflexionar sobre sus acciones en el entorno y, a la vez, que aprendan de manera autónoma utilizando los espacios naturales, realizando trabajo colaborativo entre asignaturas e integrando los contenidos desde la interdisciplinariedad (Torres, Mesina, Salamanca y Sepúlveda, 2016; Piza-Flores, 2018).

Desde esta perspectiva, los espacios naturales cobran una gran importancia para el desarrollo y enseñanza de temáticas medioambientales, constituyendo un espacio pedagógico ideal para comprender, profundizar y reflexionar sobre las interacciones que se generan en la naturaleza y cómo las acciones antrópicas generan una gran inestabilidad e, inclusive, pérdida de la biodiversidad, lo que afecta indudablemente la calidad de vida y los recursos de las futuras generaciones (y las actuales).

\section{Aulas abiertas: el oikos como tercer maestro}

Según Rousseau (2011), la naturaleza instruye mejor que el hombre y, por tanto, la mejor educación será aquella que sigue el curso de la naturaleza. A partir de esto y de las siguientes corrientes educativas relacionadas a la formación en los espacios naturales (la propuesta por la Escuela Waldorf, por ejemplo), pareciera ser que cada niño/a debiera tener la oportunidad de vivenciar el contacto con la naturaleza en uno o varios momentos de su formación, a partir del abordaje sistémico, como una forma de desarrollo de los programas educativos. La naturaleza, en su más íntima expresión, se presenta como uno de los espacios pedagógicos más potentes para aclarar conceptos de toda índole, desde lo referente al medio circundante hasta posturas filosóficas. 
La convivencia con el medio social y natural puede ser desarrollada toda vez que se promueva esta vuelta a casa, de lo contrario, se verá y entenderá siempre como dos fenómenos separados. Vale decir, la manera de comprender la realidad desde una visión globalizada y multidimensional permitiría a su vez un conocimiento y una pertenencia al percibir el entorno desde su complejidad (Castillo-Retamal, Cordero-Tapia y Scopel, 2019). En palabras de Varela (2000), cada parte actualiza el conjunto y el conjunto actualiza las partes.

Morin y Kern (1993) acuñaron el concepto de "Tierra Patria" para indicar el lugar de convivencia civilizada de la humanidad en simbiosis con el planeta, señalando la necesidad de arraigar a la humanidad en el planeta, en función de un destino complementario y errante. Es decir, este oikos es parte de todos/as y, a la vez, la humanidad es parte de él.

La problemática medioambiental, a raíz de los fenómenos hasta hoy conocidos, requiere de la participación activa y comprometida de todos los actores sociales, y que estos a su vez empapen, tiñan e impregnen sus acciones de sentido político, en el entendido de ser un "ser social" respecto al cuidado y protección medioambiental.

Sin duda, esto será posible en la medida que cada uno de esos actores comprenda la relación indisociable que existe entre ser humano y naturaleza. Más que relación en sí misma, es una condición: el ser humano es naturaleza. En este sentido, el oikos se transforma en el tercer maestro, concepto utilizado principalmente por la arquitectura y acuñado por Malaguzzi, iniciador de la metodología educativa de las escuelas Reggio Emilia (TorresLanda, 2016), donde docentes y padres/madres (o pares), son los otros dos maestros. En esta línea, el espacio como ambiente de aprendizaje se concibe como fundamental, de modo que la relación entre el proyecto pedagógico y la estructura arquitectónica debe tener cierta conexión y profundidad. Para ello, se habla de la neuroarquitectura, donde la distribución del espacio y sus materiales inciden positivamente en la adquisición de aprendizajes, a partir de la experimentación, comunicación e investigación (Torres-Landa, 2016).

Para el caso de este análisis, es posible interpretar a ese tercer maestro como el espacio natural en el que se desarrollan procesos educativos, donde la geoarquitectura, o bien, la biogeo-estructura, contribuye a la generación de aprendizajes desde su propia forma de 
exponerse. En tal sentido, Wells (2000) indica que los espacios naturales permiten vivenciar experiencias significativas, además de contribuir a la mejora de las capacidades cognitivas. Por su parte, Kellert (2008) y Fuentealba et al. (2017) sostienen que los espacios naturales son lugares especiales para la generación del conocimiento a partir de la construcción de aprendizajes.

Ahora bien, para llevar a cabo estos procesos, necesariamente los otros dos maestros deben tener algún punto de concomitancia en virtud de los objetivos de la educación ambiental. Actualmente, la formación profesional en esta área se mantiene débil y carente de posibilidades de avanzar, aunque en muchos currículos se manifiesta el abordaje de la educación ambiental de manera clara y explícita. Lamentablemente, esto repercute en los resultados que hoy vemos, principalmente desde el punto de vista de las actitudes y opiniones poco amigables y de despreocupación respecto al medio ambiente (Serrano, 2006; Serrano y Camarena, 2006; Valenzuela, 2007; Ávila y Rodríguez, 2009; Camarena, 2009; Martínez y Carballo, 2013; Ruiz y Pérez, 2014; Díaz, Camarena, Mirón y Ochoa, 2019).

En tal sentido, esta preparación de los/as docentes y el abordaje de la ducación ambiental debe atender la multidimensionalidad que posee el ser humano. Fröebel, en uno de sus principios pedagógicos, indica que las áreas de aprendizaje no pueden estar separadas, en el entendido que el propio ser humano es a su vez una unidad compleja, por tanto, integral. La adquisición de los aprendizajes debe estar en conexión con lo que se aprende y el entorno donde se desarrolla este aprendizaje, de allí la importancia de enseñar in situ lo que sucede con el medioambiente y su estado actual, haciendo carne el hecho de que lo que se conoce se quiere y lo que se quiere, se cuida (Novo y Murga, 2010).

En esta línea, conocer el espacio, visitar los ambientes naturales (artificializados o no), compartir con la esencia misma del ser humano en su hábitat natural, probablemente contribuya a generar actitudes pro-ambientales, desde el conocimiento más que desde el activismo. Cuidar el oikos como parte de la propia existencia es hoy la tarea desafiante que nos presenta el estado actual del mismo, condición que sin lugar a dudas podría verse aminorada al incluir al tercer maestro en las acciones pedagógicas como eje transversal de los procesos educativos. 


\section{Consideraciones finales}

La naturaleza y la educación, al igual que todo sistema complejo, requieren un tratamiento y visión que dé cuenta de una mirada holística sobre ambos, para atender de igual forma las necesidades que presentan. Para ello, es necesario contar con profesionales de la educación que presenten características asociadas a la tarea, vale decir, profesionales integrales que entreguen una formación integral. Hoy día es posible ver en los currículos escolares la instauración de la educación ambiental como parte de un lineamiento transversal, con el que se pretende construir valores, potenciar actitudes y establecer relaciones tendientes a cuidar el medioambiente. Sin embargo, lo que se diseña en el currículo no siempre se condice con las acciones de quienes lo administran.

Al mismo tiempo, esta falta de preparación y posible desinterés, no permiten un avance con la rapidez que se necesita. La desinformación y repetición de patrones en educación obnubilan por momentos los objetivos y las acciones de quienes han conseguido adelantar algún paso en la materia. Sin embargo, en la medida que la preparación de los profesionales para atender a los nuevos currículos se ajuste a la necesidad sistémica de su abordaje, se estará dando un paso en la mejora actitudinal frente al fenómeno que está afectado las aulas abiertas y naturales, el oikos.

Para ello es necesario redoblar los esfuerzos, lo que a su vez requiere voluntad política desde el punto de vista del ser social, toda vez que al valorizar la materia viva nos acercamos al encuentro con el propio origen humano. El contacto con la naturaleza, en todas sus manifestaciones, nos retrotrae a la esencia del ser, al inicio de la vida, al encuentro de las respuestas y al descubrimiento de los secretos; es decir, el entorno se transforma en espacio y maestro, en el entendido de mostrar la raíz para la explicación del fenómeno propio de la existencia. 
REVISTA SABERES EDUCATIVOS, N 4, ENERO-JUNIO 2020

\section{Referencias}

Agüero, B., Matus, M., Lillo, D., Marfull, R., Uribe, D., Valdivia, R \& Zamorano, D. (2011). Educación Ambiental en Chile, falencias y desafíos. Artículo en preparación.

Ávila, M. \& Rodríguez, M. (2009). Capacitación, formación y profesionalización en educación ambiental: tres alternativas diferenciadas y complementarias. Memorias del IX Congreso Nacional de Investigación Educativa. Mérida, México. Recuperado de http://www.comie.org.mx/congreso/memoriaelectronica/v10/pdf/area_tematica_03/pon encias/1041-F.pdf

Camarena, B. (2009). Educación ambiental y formación de profesorado en México: estudio de perfiles ambientales en las escuelas normales de Sonora (Tesis Doctoral en Educación). Universidad de Salamanca, España.

Castillo-Retamal, F. y Cordero-Tapia, F. (2019). La educación ambiental en la formación de profesores en Chile. UC Maule, 56(1), 9-28. Recuperado de http://revistaucmaule.ucm.cl/article/view/66

Castillo-Retamal, F., Cordero-Tapia, F. y Scopel, A.J. (2019). Interdisciplina y educación: la orientación deportiva como propuesta sistémica. Revista Pensar en Movimiento, 17(2), s/p. DOI: 10.15517/pensarmov.v17i2.36693

Castillo-Sarmiento, A. Y., Suárez-Gélvez, J. H., y Mosquera-Téllez, J. (2017). Naturaleza y sociedad: relaciones y tendencias desde un enfoque eurocéntrico. Luna Azul, 44, s/p. DOI: 10.17151/luaz.2017.44.21

Ley $N^{\circ}$ 20.370. Diario Oficial de la República de Chile, Santiago, Chile, 12 de septiembre de 2009.

Decreto Supremo $N^{\circ} 433$, Establece las Bases Curriculares para la Educación Básica en las Asignaturas que Indica. Diario Oficial de la República de Chile, Santiago, Chile, 19 de diciembre de 2012.

Ministerio del Medio Ambiente, Gobierno de Chile. (2018a). Educación ambiental para la sustentabilidad: sintesis para el docente. Recuperado de https://mma.gob.cl/wpcontent/uploads/2018/08/Guia-Docentes-EA_web.pdf 
EL TERCER MAESTRO: EL ESPACIO NATURAL COMO CATALIZADOR PARA UNA EDUCACIÓN

AMBIENTAL EFECTIVA / CASTILLO-RETAMAL; CORDERO-TAPIA

Ministerio del Medio Ambiente, Gobierno de Chile. (2018b). Educación Ambiental. Una mirada desde la institucionalidad ambiental chilena. Recuperado de https://mma.gob.cl/wpcontent/uploads/2018/08/LIBRO-EDUCACION-AMBIENTAL-final_web.pdf

Díaz, G., Camarena, B., Mirón, C. \& Ochoa, E. (2019). Prácticas docentes en educación ambiental y habilidades proambientales en el estudiantado de quinto grado de primaria. Revista Actualidades Investigativas en Educación, 19(3), 1-18. DOI: https://doi.org/10.15517/aie.v19i3.38797

Eslava-Zapata, R., Zambrano-Vivas, M., Chacón-Guerrero, E., González-Júnior, H. \& MartínezNieto, A. Estrategias didácticas para la promoción de valores ambientales en la educación primaria. Aibi, 6(2), 24-72. DOI: https://doi.org/10.15649/2346030X.476

Frankl, V. (2000). En el principio era el sentido, reflexiones en torno al ser humano. Buenos Aires, Argentina: Paidós.

Fuentealba, M. (2011). Reflexión sobre impacto ambiental antrópico, desarrollo sustentable y educación ambiental. UC Maule, 41, 29-43. Recuperado de http://repositorio.ucm.cl/handle/ucm/1255

Fuentealba, M. (2018). Valoración actitudinal proambiental: un análisis global en estudiantes de enseñanza primaria, secundaria y terciaria. Luna Azul, 47, 159-176. Recuperado de http://vip.ucaldas.edu.co/lunazul/downloads/Lunazul47_9.pdf

Fuentealba, M., Marín, F., Castillo, F. y Roco, L. (2017). Análisis de la experiencia pedagógica: Campamento Explora Chile Va! Valorando la biodiversidad maulina. Actualidades Investigativas en Educación, 17(1), 1-25. DOI: http://dx.doi.org/10.15517/aie.v17i1.27211

Delors, J. (2010). La Educación encierra un tesoro. Informe a la UNESCO de la Comisión Internacional sobre la Educación para el Siglo XXI (compendio). Madrid/París: Santillana/UNESCO.

Juyent, M. y Kong, F. (2011). Envisioning the Future: A Case Study in a Primary School in Barcelona. Eingana, 34(3), 16-23. Recuperado de https://grupcomplex.uab.cat/_docs/Junyent_Kong_2011_Envisioning_the_future.pdf 
REVISTA SABERES EDUCATIVOS, N 4, ENERO-JUNIO 2020

Kellert, S. (2008). Building for Life: Designing and Understanding the Human-Nature Connection. Washington, Estados Unidos: Island Press.

Leff, E. (2006). Aventuras de la epistemología ambiental: de la articulación de las ciencias al diálogo de saberes. Ciudad de México: Siglo XXI.

Martínez, M. y Carballo, L. (2013). La educación ambiental rural desde las escuelas básicas y por estas. Revista Electrónica Educare, 17(2), 69-79. Recuperado de https://www.scielo.sa.cr/pdf/ree/v17n2/a05v17n2.pdf

Mejía, M. (2016). Una educación ambiental desde la perspectiva cultural para la formación de profesores de ciencias naturales. Luna Azul, 43, 354-385. Recuperado de http://www.scielo.org.co/pdf/luaz/n43/n43a16.pdf

Mendieta, M. y Gutiérrez, G. (2014). Actitudes ambientales hacia el agua, una exploración en estudiantes del municipio de Ventaquemada (Boyacá). Luna Azul, 39, 40-62. Recuperado de http://ref.scielo.org/p8ykzk

Montserrat, J. (2019). El hombre y la naturaleza. Revista Pensamiento, 75(283), 3-4. Recuperado de https://revistas.comillas.edu/index.php/pensamiento/article/viewFile/11312/10651

Morin, E. \& Kern, A. (1993). Tierra Patria. Buenos Aires, Argentina: Nueva Visión.

Muñoz-Pedreros, A. (2014). La educación ambiental en Chile, una tarea aún pendiente. Ambiente \& Sociedade, 17(3), 177-198. Recuperado de http://www.scielo.br/pdf/asoc/v17n3/v17n3a11.pdf

Novo, M. \& Murga, M. (2010). Educación Ambiental y Ciudadanía Planetaria. Revista Eureka 7,(N $\quad$ Extraordinario), 179-186. Recuperado de https://www.redalyc.org/pdf/920/92013009003.pdf

Pérez, M. Á, Pérez, M. y Quijano, R. (2009). Valoración del cambio de actitudes hacia el medio ambiente producido por el programa didáctico "EICEA" en los alumnos de educación secundaria obligatoria (14-16 años). Revista Electrónica de Enseñanza de las Ciencias, 8(3), 1019-1036. Recuperado de http://reec.uvigo.es/volumenes/volumen8/ART14_Vol8_N3.pdf 
Piza-Flores, V. (2018). Transversalidad del eje "medio ambiente" en educación superior: un diagnóstico de la Licenciatura en Contaduría de la UAGro. Revista Iberoamericana para la Investigación y el Desarrollo Educativo, 8(16), 1-24. DOI: http://dx.doi.org/10.23913/ride.v8i16.360

Castillo, F. (2011). El parque como aula: configuración escenográfica para el aprendizaje con sentido. En J. Suárez (Ed.): Recreación: el parque como encuentro de desarrollo social y ciudadano (10-19). Medellín, Colombia: Funámbulos Editores. Recuperado de http://viref.udea.edu.co/contenido/publicaciones/libros_expo2011/recreacion_parqup_c omo\%20encuentro_desarrollo_social_ciudadano.pdf

Rousseau, J. (2011). Emilio o de la Educación. Madrid, España: Alianza Editorial.

Ruiz, M. y Pérez, E. (2014). Educación ambiental en niños y niñas de instituciones educativas oficiales del distrito de Santa Marta. Zona próxima, 21, 52-64. Recuperado de http://www.scielo.org.co/scielo.php?script=sci_abstract\&pid=S214594442014000200005

Sauvé, L. (2004). Una cartografía de corrientes en educación ambiental. En M. Sato \& I. Calvalho, I. (Eds.). A pesquisa em educação ambiental: cartografias de uma identidade narrativa em formação. Porto Alegre, Brasil: Artmed. Recuperado de https://www.scienceopen.com/document?vid=5295a56c-469e-4063-bd010de672cb058b

Serrano, A. (2006). Educación ambiental en las escuelas primarias: conocimientos y barreras institucionales desde la perspectiva docente (Tesis de Maestría en Desarrollo Regional). Centro de Investigación en Alimentación y Desarrollo, Sonora, México.

Serrano, A. y Camarena, B. (2006). Docentes y barreras institucionales a la educación ambiental. Memorias del VIII Congreso Nacional de Investigación Educativa. Hermosillo, México. Recuperado de http://www.comie.org.mx/congreso/memoriaelectronica/v09/ponencias/at03/PRE11781 27475.pdf 
Sosa, S., Isaac-Márquez, R., Eastmond, A., Ayala, M. y Arteaga, M. (2010). Educación superior y cultura ambiental en el sureste de México. Revista Universidad y Ciencia, 26(1), 3349. Recuperado de http://www.scielo.org.mx/scielo.php?script=sci_arttext\&pid=S018629792010000100003

Strobl, G. (2005). Una educación ambiental conforme a la época. Revista de Educación Ambiental, 5, 48-50.

Tikka, P., Kuitunen, M. \& Tynys, S. (2000). Effects of Educational Background on Students Attitudes, Activity Levels, and Knowledge Concerning the Environment. Journal of Environmental Education, $31(3), \quad 12-19 . \quad$ DOI: https://doi.org/10.1080/00958960009598640

Torres-Landa, A. (2016). “El Tercer Maestro” para el siglo XXI. Aguas Calientes, México: Editorial Universidad Autónoma de Aguas Calientes.

Torres, L., Mesina, N., Salamanca, B y Sepúlveda, C. (2016). Efectos de la enseñanza interdisciplinaria en la educación ambiental sobre los conocimientos, valores y actitudes ambientales de estudiantes de segundo ciclo básico (Los Ángeles, Región del Biobío, Chile). Revista Complutense de Educación, 27(3), 1139-1155. DOI: https://doi.org/10.5209/rev_RCED.2016.v27.n3.47551

Tréllez, E. (2006). Educación ambiental y sostenibilidad política: democracia y participación. Revista Polis, 14, 1-14. Recuperado de https://journals.openedition.org/polis/5167

Valenzuela, B. (2007). Variables disposicionales promotoras de la conducta proambiental en estudiantes de sexto grado de primaria (Tesis de Maestría en Innovación Educativa). Universidad de Sonora, México.

Varela, F. (2000). El fenómeno de la vida. Santiago, Chile: Dolmen.

Vendrasco, N., Guzmán, E., Carrasco, X., y Merino, C. (2018). 7A010 Formación de profesores en Educación Ambiental: una experiencia en Chile. Revista Tecné, Episteme y Didaxis, ( $\mathrm{N}^{\mathrm{o}}$ Extraordinario), $1-8 . \quad$ Recuperado de http://revistas.pedagogica.edu.co/index.php/TED/article/view/9112 
EL TERCER MAESTRO: EL ESPACIO NATURAL COMO CATALIZADOR PARA UNA EDUCACIÓN AMBIENTAL EFECTIVA / CASTILLO-RETAMAL; CORDERO-TAPIA

Wells, N. (2000). At Home with Nature: Effects of "Greenness" on Children's Cognitive Functioning. Journal Environment and Behavior, 32 (6), 775-795. DOI: https://doi.org/10.1177/00139160021972793 